Génét. Sél. Evol., 1985, 17 (4), 561-578

\title{
Mise au point
}

\section{Interactions géniteur $\times$ population des partenaires III. Synthèse bibliographique}

\author{
J.M. BRUN \\ I.N.R.A., Station d'Amélioration Génétique des Animaux \\ B.P. 27, F 31326 Castanet-Tolosan Cedex
}

\begin{abstract}
Résumé
Une analyse bibliographique de résultats expérimentaux sur espèces animales a été entreprise pour tester l'existence et étudier le déterminisme des interactions qui peuvent exister, sur un caractère quantitatif, lorsque des géniteurs d'une population donnée se reproduisent d'une part intra-population et d'autre part en croisement avec une autre population. Elles peuvent se traduire par une différence de classements sur descendance des géniteurs ou encore par une différence de variabilité des descendances selon le système de reproduction.

L'approche du phénomène est double et consiste à analyser d'une part les paramètres statistiques estimés de l'interaction (corrélation génétique pur-croisement, héritabilités en pur et en croisement) et d'autre part, des paramètres " réalisés " de cette interaction, lorsqu'elle s'exprime de façon dynamique dans des expériences de sélection à 2 populations (sélection intra-population et sélection récurrente réciproque). Ces 2 approches concordent pour indiquer que ces phénomènes se rencontrent surtout pour des caractères à faible héritabilité, présentant de l'hétérosis (caractères d'adaptation) et cela d'autant plus que leur passé de sélection est ancien. Ces interactions se traduisent alors par des valeurs faibles, voire négatives, de la corrélation génétique pur-croisement et une différence de variabilité additive en faveur du croisement. Cependant, des interactions peuvent également apparaître sur des caractères pondéraux, chez les mammifères polytoques notamment, avec parfois une réduction de la variabilité génétique additive en croisement. On propose une hypothèse pour rendre compte des interactions sur les caractères d'adaptation, basée sur l'existence d'une liaison non linéaire entre les effets additifs d'un géniteur intrapopulation et en croisement.
\end{abstract}

Mots clés : Interactions génotype $\times$ milieu, corrélation génétique pur-croisement, héritabilité en crcisement, sélection récurrente et réciproque.

\section{Summary}

"Breeding animal " $\times$ " mate population " interactions.

III. A review

The objective of this review is to test for the existence and study the mechanisms of the interactions which may exist between the genotype of a sire and the population of its mates, in the case of pure and crossbreeding : they may lie in differences in ranks for the progeny tested sires 
and in differences in the variability of their progeny performances according to the breeding system. Two types of indicators are analysed: estimated parameters (genetic correlation between pure and crossbred performance $\left(r_{p c}\right)$, pure and crossbred heritabilities) and « realised " parameters calculated from selection experiments involving 2 populations (intra-population or reciprocal recurrent selection). These 2 approaches indicate that such interactions are more likely to occur for heterotic traits having a low heritability (such as fitness traits) and the longer they are selected for. In this case, the interactions result in low or negative values of $r g_{p c}$ and in a higher crossbred heritability. However, interactions can also affect production traits, particularly in polytokous mammals, with a possible reduction of the additive genetic variability in the crossbreds. So as to explain the interactions observed on fitness traits, the curvilinear relationship between the additive effects of a sire in pure and in crossbreding is proposed as an hypothesis.

Key words: Genotype environment interactions, genetic correlation between purebred and crossbred performance, crossbred heritability, reciprocal recurrent selection.

\section{Introduction}

Les facteurs de milieu susceptibles de modifier l'expression phénotypique d'une différence génétique donnée - et ainsi produire des interactions génotype $\times$ milieu peuvent être d'ordre physique, économique ou encore biologique (DICKERSON, 1962). La question du choix d'une méthode de sélection d'une souche pour améliorer ses résultats en croisement peut s'interpréter en termes d'interactions génotype $\times$ milieu, le terme de " génotype » désignant alors le génotype d'un géniteur et celui de « milieu », le type génétique de ses partenaires, c'est-à-dire, sa population partenaire (BRUN, 1982, 1984). Ces interactions, s'exprimant sur un caractère quantitatif, se mesurent sur les descendants des géniteurs considérés. Conformément à la classification générale de Bowman (1972), elles peuvent se présenter de 2 façons : la première est un changement du classement des géniteurs sur descendance selon la population partenaire, la deuxième, une différence de variabilité de leurs descendances selon la population partenaire.

Ces interactions s'expriment également de manière dynamique dans des processus de sélection lorsqu'une population est sélectionnée dans un "milieu génétique " donné et la réponse mesurée dans un autre «milieu génétique », comme par exemple la réponse obtenue en croisement à une sélection intra-population.

L'objectif de cette revue bibliographique est d'étudier ces phénomènes dans les espèces animales domestiques, en se limitant au cas où les géniteurs d'une population donnée sont confrontés à 2 populations partenaires, leur population d'origine et une autre population. La rareté des publications exploitables dans ce sens a conduit à retenir également les résultats concernant des animaux de laboratoire (Drosophile, Tribolium, Souris) considérés comme modèles biologiques pour les espèces zootechniques et à n'écarter aucun caractère quantitatif.

On a préalablement défini 2 types d'indicateurs de ces interactions : les premiers sont des paramètres " estimés ", les paramètres statistiques qui caractérisent l'interaction s'exprimant à une génération donnée (BRUN, 1982). Les seconds sont des paramètres " réalisés " (cf. concept d' " héritabilité réalisée ») qui tentent d'appréhender l'expression dynamique de ces interactions (BRUN, 1984). Cette publication se ramène donc à l'analyse de la variation de ces 2 types d'indicateurs. 


\section{Analyse des paramètres estimés de l'interaction géniteur $\times$ population partenaire}

\section{A. Paramètres analysés et méthode d'analyse}

Les paramètres proposés comme indicateurs théoriques des 2 modes d'expression de l'interaction (différences de classements et de variabilités) sont respectivement :

$$
r\left(\Gamma_{p}, \Gamma_{c}\right) \text { et le couple } v\left(\Gamma_{p}\right), v\left(\Gamma_{c}\right)
$$

où $\Gamma_{p}$ (resp. $\Gamma_{c}$ ) désigne l'aptitude générale à la combinaison (ou encore effet additif) d'un géniteur, intra-population (resp. en croisement), $r$, une corrélation et $v$, une variance. Si l'estimation du premier paramètre est généralement fournie par les auteurs, les 2 derniers sont rarement publiés. A leur place, on analysera les paramètres $h_{p}^{2}$ et $h_{c}^{2}$, expressions relatives des variabilités de $\Gamma_{p}$ et de $\Gamma_{c}$ :

$$
h_{p}^{2}=4 v\left(\Gamma_{p}\right) / v\left(\mathrm{Y}_{\mathrm{p}}\right) \text { et } h_{c}^{2}=4 v\left(\Gamma_{c}\right) / v\left(\mathrm{Y}_{c}\right)
$$

où $\mathrm{Y}_{p}$ et $\mathrm{Y}_{c}$ désignent la valeur phénotypique des descendants respectivement purs et croisés des géniteurs considérés. Dans la suite, on désignera $r\left(\Gamma_{p}, \Gamma_{c}\right)$ par le terme de «corrélation génétique pur-croisement » (en le notant $r g_{p c}$ ) et le couple $\left(h_{p}^{2}, h_{c}^{2}\right)$ par héritabilité en pur et en croisement.

Les modèles statistiques d'estimation de ces paramètres sont des analyses de variance hiérarchiques à effets aléatoires, appliquées séparément à la population de produits purs et croisés d'un échantillon de mâles d'une population donnée. A un couple de populations correspondent en général dans les publications 2 séries de tels paramètres, une pour chaque population d'origine des géniteurs. Il existe cependant des cas où les auteurs n'en donnent qu'une seule série, estimés sur l'ensemble des 2 populations. La structure de l'information par espèce et par caractère présente un fort déséquilibre et de nombreuses cellules ne sont représentées que par une seule expérience (tabl. 1). En outre, la variabilité des résultats semble différer selon l'espèce et le caractère considéré. La structure des données se prête donc mal à une analyse statistique globale de la variation des paramètres en fonction de l'espèce et du caractère, du type d'analyse de variance à 2 facteurs. En conséquence, on analysera cette variation en plusieurs temps : après une étude d'ensemble des estimées, on examinera les résultats par groupe d'espèces, d'abord globalement, puis en fonction du caractère. On abordera ensuite l'étude des variations dans le cadre d'une association espèce-caractère. On analysera séparément les 2 indicateurs de l'interaction, c'est-à-dire, d'une part les rapports entre héritabilité en pur et en croisement et d'autre part, la corrélation génétique pur-croisement et ses variations en liaison avec $h_{p}^{2}$. Notons ici le double statut de $h_{p}^{2}$ : associé à $h_{c}^{2}$, c'est l'un des 2 paramètres de l'interaction (également appelé "indicateur") ; c'est aussi un paramètre que l'on teste comme facteur de variation de l'interaction.

Pour l'étude des liaisons entre paramètres, on a réalisé dans certains cas des ajustements polynomiaux (de $h_{c}^{2}$ sur $h_{p}^{2}$ et de $r g_{p c}$ sur $h_{p}^{2}$ ), en introduisant les valeurs brutes des paramètres, c'est-à-dire sans tenir compte de leur précision qui n'est pas mentionnée par tous les auteurs. Dans l'étude de la liaison entre $r g_{p c}$ et $h_{p}^{2}$, les valeurs manquantes du dernier paramètre sont remplacées par sa valeur moyenne pour l'espèce et le caractère considérés. Dans cette recherche de mise en évidence de tendances générales, on n'a pas réalisé de test de signification statistique compte tenu du niveau de l'information actuellement disponible sur la loi statistique des paramètres étudiés. 
TABLEAU 1

Structure de l'information sur les paramètres de l'interaction géniteur $\times$ population partenaire en fonction de l'espèce et du caractère.

Structure of the information about the parameters of the interaction between breeding animal and population of mates according to the species and traits.

\begin{tabular}{|c|c|c|c|c|c|c|c|c|}
\hline \multirow[b]{2}{*}{ Espèce } & \multirow[b]{2}{*}{ Caractère } & \multicolumn{4}{|c|}{$\begin{array}{l}\text { Héritabilités en pur } \\
\text { et en croisement } \\
\left(h_{p}^{2} \text { et } h_{c}^{2}\right)\end{array}$} & \multicolumn{3}{|c|}{$\begin{array}{l}\text { Corrélation génétique } \\
\text { pur-croisement } \\
\qquad g_{p c}\end{array}$} \\
\hline & & $n_{e}$ & $n_{h}$ & $h_{p}^{2}$ & $h_{c}^{2}$ & $n_{e}$ & $n_{r g}$ & $r g_{p c}$ \\
\hline Poule & $\begin{array}{l}\text { Ponte } \ldots \ldots \ldots \\
\text { Age au } 1^{\text {er }} \text { œuf } \ldots \ldots \ldots \ldots \ldots \\
\text { Poids corporel } \ldots \ldots \ldots \ldots \ldots \\
\text { Poids de l'œuf } \ldots \ldots \ldots \ldots \ldots\end{array}$ & $\begin{array}{l}8 \\
3 \\
4 \\
5\end{array}$ & $\begin{array}{r}18 \\
6 \\
8 \\
12\end{array}$ & $\begin{array}{l}0,17 \\
0,46 \\
0,47 \\
0,52\end{array}$ & $\begin{array}{l}0,24 \\
0,46 \\
0,54 \\
0,54\end{array}$ & $\begin{array}{l}7 \\
4 \\
3 \\
4\end{array}$ & $\begin{array}{r}16 \\
8 \\
6 \\
10\end{array}$ & $\begin{array}{l}0,45 \\
0,68 \\
0,85 \\
0,76\end{array}$ \\
\hline Souris & $\begin{array}{l}\text { Caractères de la portée } \ldots \ldots \ldots \\
\text { Poids à la naissance } \ldots \ldots \ldots \\
\text { Poids au sevrage } \ldots \ldots \ldots \ldots \\
\text { Poids post-sevrage } \ldots \ldots \ldots \ldots\end{array}$ & $\begin{array}{l}1 \\
1 \\
1 \\
1\end{array}$ & $\begin{array}{l}2 \\
2 \\
1 \\
6\end{array}$ & $\begin{array}{l}0,40 \\
0,25 \\
0,00 \\
0,45\end{array}$ & $\begin{array}{l}0,62 \\
0,20 \\
0,32 \\
0,25\end{array}$ & $\begin{array}{l}1 \\
1 \\
0 \\
1\end{array}$ & $\begin{array}{l}2 \\
2 \\
0 \\
6\end{array}$ & $\begin{array}{c}0,65 \\
0,61 \\
\overline{1,04}\end{array}$ \\
\hline Porc & $\begin{array}{l}\text { Caractères de la portée } \ldots \ldots \ldots \\
\text { Poids à la naissance } \ldots \ldots \ldots \\
\text { Poids au sevrage } \ldots \ldots \ldots \ldots \\
\text { Poids post-sevrage } \ldots \ldots \ldots \\
\text { Epaisseur du lard } \ldots \ldots \ldots \ldots\end{array}$ & $\begin{array}{l}0 \\
1 \\
2 \\
3 \\
3\end{array}$ & $\begin{array}{l}0 \\
2 \\
3 \\
5 \\
5\end{array}$ & $\begin{array}{l}- \\
0,18 \\
0,13 \\
0,44 \\
0,49\end{array}$ & $\begin{array}{l}- \\
0,05 \\
0,44 \\
0,22 \\
0,42\end{array}$ & $\begin{array}{l}2 \\
1 \\
1 \\
3 \\
3\end{array}$ & $\begin{array}{l}3 \\
1 \\
1 \\
5 \\
4\end{array}$ & $\begin{array}{l}0,54 \\
0,70 \\
0,83 \\
0,60 \\
0,76\end{array}$ \\
\hline Mouton & $\begin{array}{l}\text { Poids à la naissance } \ldots \ldots \ldots \\
\text { Poids au sevrage } \ldots \ldots \ldots\end{array}$ & $\begin{array}{l}1 \\
2\end{array}$ & $\begin{array}{l}2 \\
3\end{array}$ & $\begin{array}{l}0,60 \\
0,37\end{array}$ & $\begin{array}{l}0,14 \\
0,27\end{array}$ & $\begin{array}{l}0 \\
1\end{array}$ & $\begin{array}{l}0 \\
1\end{array}$ & $\overline{0,82}$ \\
\hline Boeuf & $\begin{array}{l}\text { Poids à la naissance } \ldots \ldots \ldots \\
\text { Poids au sevrage } \ldots \ldots \ldots \ldots \\
\text { Poids post-sevrage } \ldots \ldots \ldots \\
\text { Etat d'engraissement } \ldots \ldots \ldots\end{array}$ & $\begin{array}{l}2 \\
2 \\
2 \\
1\end{array}$ & $\begin{array}{l}5 \\
5 \\
3 \\
1\end{array}$ & $\begin{array}{l}0,57 \\
0,43 \\
0,54 \\
0,39\end{array}$ & $\begin{array}{l}0,47 \\
0,43 \\
0,66 \\
0,54\end{array}$ & $\begin{array}{l}2 \\
2 \\
2 \\
1\end{array}$ & $\begin{array}{l}6 \\
6 \\
3 \\
1\end{array}$ & $\begin{array}{l}0,91 \\
1,02 \\
0,62 \\
0,37\end{array}$ \\
\hline Tribolium & $\begin{array}{l}\text { Ponte } \ldots \ldots \ldots \ldots \ldots \ldots \\
\text { Poids pupal } \ldots \ldots \ldots \ldots \ldots\end{array}$ & $\begin{array}{l}1 \\
1\end{array}$ & $\begin{array}{l}3 \\
2\end{array}$ & $\begin{array}{l}0,24 \\
0,18\end{array}$ & $\begin{array}{l}0,32 \\
0,32\end{array}$ & $\begin{array}{l}0 \\
1\end{array}$ & $\begin{array}{l}0 \\
1\end{array}$ & $\overline{0,40}$ \\
\hline Drosophile & Ponte $\ldots \ldots \ldots \ldots \ldots$ & 2 & 4 & 0,05 & 0,11 & 1 & 2 & $-0,58$ \\
\hline Ensemble & & & 98 & 0,35 & 0,36 & & 81 & 0,68 \\
\hline
\end{tabular}

$n_{e}$ est le nombre d'expériences ; $n_{h}$ est le nombre d'estimées du couple $\left(h_{p}^{2}, h_{c}^{2}\right) ; n_{r g}$ est le nombre d'estimées de $r g_{p r}$

\section{B. Variations entre espèces et caractères des indicateurs de l'interaction}

\section{Valeurs relatives de $h_{p}^{2}$ et $h_{c}^{2}$}

Sur l'ensemble des résultats, on note des différences entre les estimées d'héritabilité en pur et en croisement (fig. 1). Si la signification statistique de ces différences au niveau de chaque expérience n'est que rarement testée par les auteurs, on note 
cependant une tendance à la supériorité de l'héritabilité en croisement pour les faibles valeurs de $h_{p}^{2}$ et la tendance inverse pour les valeurs de $h_{p}^{2}$ dépassant 0,4 . Dans la grande majorité des cas, il n'est pas possible de savoir si la différence observée relève d'une différence de variabilité additive de l'effet géniteur selon la population partenaire (numérateur de $h^{2}$ ) ou bien d'une différence portant sur le dénominateur, c'est-à-dire, sur la variabilité phénotypique des populations de produits purs et croisés.

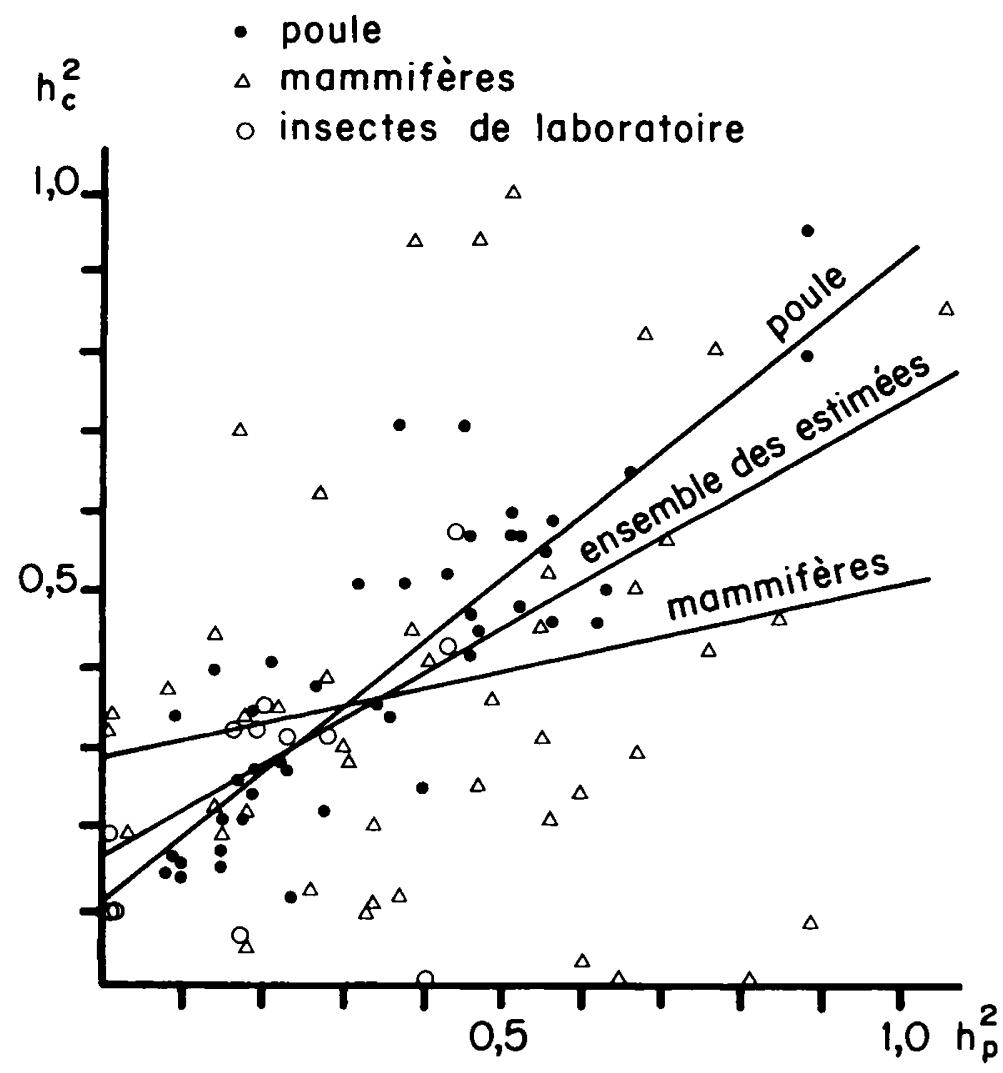

Figure 1

Valeurs relatives des héritabilités en pur $\left(\mathrm{h}_{\mathrm{p}}^{2}\right)$ et en croisement $\left(\mathrm{h}_{\mathrm{\jmath}}^{2}\right)$.

Relative values of the purebred and crossbred heritabilities $\left(\mathrm{h}_{\mathrm{p}}^{2}\right.$ and $\mathrm{h}_{\mathrm{c}}^{2}$ resp.).

Chez la poule, l'intensité de la liaison linéaire entre $h_{p}^{2}$ et $h_{c}^{2}$ est plus forte que dans l'ensemble des espèces et la droite d'ajustement de $h_{c}^{2}$ en $h_{p}^{2}$ y est plus proche de la droite d'équation $h_{c}^{2}=h_{p}^{2}$ (fig. 1). On retrouve cependant la tendance à la supériorité de $h_{c}^{2}$ sur $h_{p}^{2}$ pour les faibles valeurs de $h_{p}^{2}$, en l'occurrence pour la ponte, où le couple $\left(h_{p}^{2} / h_{c}^{2}\right)$ prend la valeur moyenne de $(0,17 / 0,24)$. Les 3 autres caractères étudiés chez la poule (âge au $1^{\text {er }}$ œuf, poids de la poule, poids de l'œuf), dont l'héritabilité en pur est voisine de 0,50 , présentent moins de différence relative entre $h_{p}^{2}$ et $h_{c}^{2}$ (tabl. 1). 
Par contre, sur l'ensemble des caractères étudiés chez les mammifères, il ne se dégage pas nettement de relations entre l'héritabilité en pur et en croisement. La droite d'ajustement linéaire de $h_{c}^{2}$ sur $h_{p}^{2}$, à faible coefficient de détermination, présente un basculement par rapport à celle tracée pour la poule (fig. 1). De fait, de nombreuses situations à forte héritabilité intra-population se caractérisent par une supériorité de $h_{p}^{2}$ sur $h_{c}^{2}$. Pour les caractères à faible héritabilité, on retrouve la tendance déjà observée. Les 2 seules estimées concernant les caractères de reproduction (obtenues chez la souris) favorisent l'héritabilité en croisement. Les caractères liés à la croissance des jeunes présentent ici des différences d'héritabilités selon le système de reproduction (tabl. 1) :

Pour le poids individuel à la naissance, ces différences favorisent $h_{p}^{2}$ aussi bien dans les espèces polytoques (porc, souris) que chez le mouton et le bœuf. Dans l'ensemble des espèces, on note une tendance à l'inversion des rapports entre héritabilités en pur et en croisement entre la naissance et le sevrage et singulièrement chez la souris et le porc. Sur ces dernières espèces, on enregistre encore d'importantes différences entre $h_{p}^{2}$ et $h_{c}^{2}$ sur les caractères pondéraux après le sevrage en faveur de $h_{p}^{2}$. Concernant les caractères liés à la composition tissulaire de la carcasse, les 3 résultats relatifs au porc (épaisseur du lard dorsal) montrent peu de différence moyenne entre les héritabilités en pur et en croisement $(0,49$ et 0,42 respectivement), et la seule observation concernant l'état de gras de la carcasse chez les bovins $(0,39 ; 0,54)$ ne correspond pas à une différence significative.

\section{Valeurs de la corrélation génétique pur-croisement}

La tendance des corrélations génétiques pur-croisement est exprimée à la figure 2 par leur courbe d'ajustement polynomial en fonction des valeurs d'héritabilité en pur : pour les valeurs de $h_{p}^{2}$ supérieures à $0,3, r g_{p c}$ présente une moyenne relativement stable, de l'ordre de 0,75 ; par contre, lorsque l'héritabilité en pur passe de 0,3 à 0,0 , la corrélation génétique pur-croisement décroît rapidement : sa valeur ajustée est de 0,57 pour $h_{p}^{2}=0,20$ et 0,45 pour $h_{p}^{2}=0,10$. Les valeurs négatives de $r g_{p c}$ sont rares. On note la possibilité de valeurs faibles pour des caractères à forte héritabilité.

Chez la poule la corrélation génétique pur-croisement discrimine la ponte, où $\mathrm{rg}_{p c}$ est égale à 0,45 en moyenne, de l'ensemble des trois autres caractères, où les valeurs de $r_{p c}$ sont plus fortes, avec une moyenne de 0,75 . La dispersion des valeurs de ce paramètre est maximale pour la ponte où ce paramètre varie entre $-0,35$ et $+1,10$.

Chez les mammifères, il ne se dégage pas de tendance nette pour $r g_{p c}$ en fonction de $h_{p}^{2}$. On remarque l'absence d'estimées faibles ou négatives pour les faibles valeurs de l'héritabilité en pur, qui pourrait être un biais dû à la rareté des estimations de $r g_{p c}$ pour les caractères de reproduction. Pour ces derniers, les estimations de la covariance génétique pur-croisement (dont le signe est celui de $r g_{p c}$ ) fournissent en effet des valeurs négatives : 15 sur les 25 observations relatives aux tailles et poids de portée chez le porc et la souris. Pour les caractères de croissance, les valeurs moyennes de $r g_{p c}$ sont élevées, quels que soient l'espèce et le caractère considérés (tabl. 1), indiquant de faibles différences de classement des géniteurs en pur et en croisement. On relève cependant des cas de corrélations pur-croisement inférieures à 0,5 voire négatives. Les critères de l'état d'engraissement offrent des valeurs élevées en moyenne $(0,76$ chez le porc en moyenne sur 4 expériences avec une gamme de variation assez large, comprise entre 0,20 et une valeur non fournie supérieure à 1). La seule estimée concernant les bovins est de $0,37 \pm 0,30$ pour l'état de gras de la carcasse. 


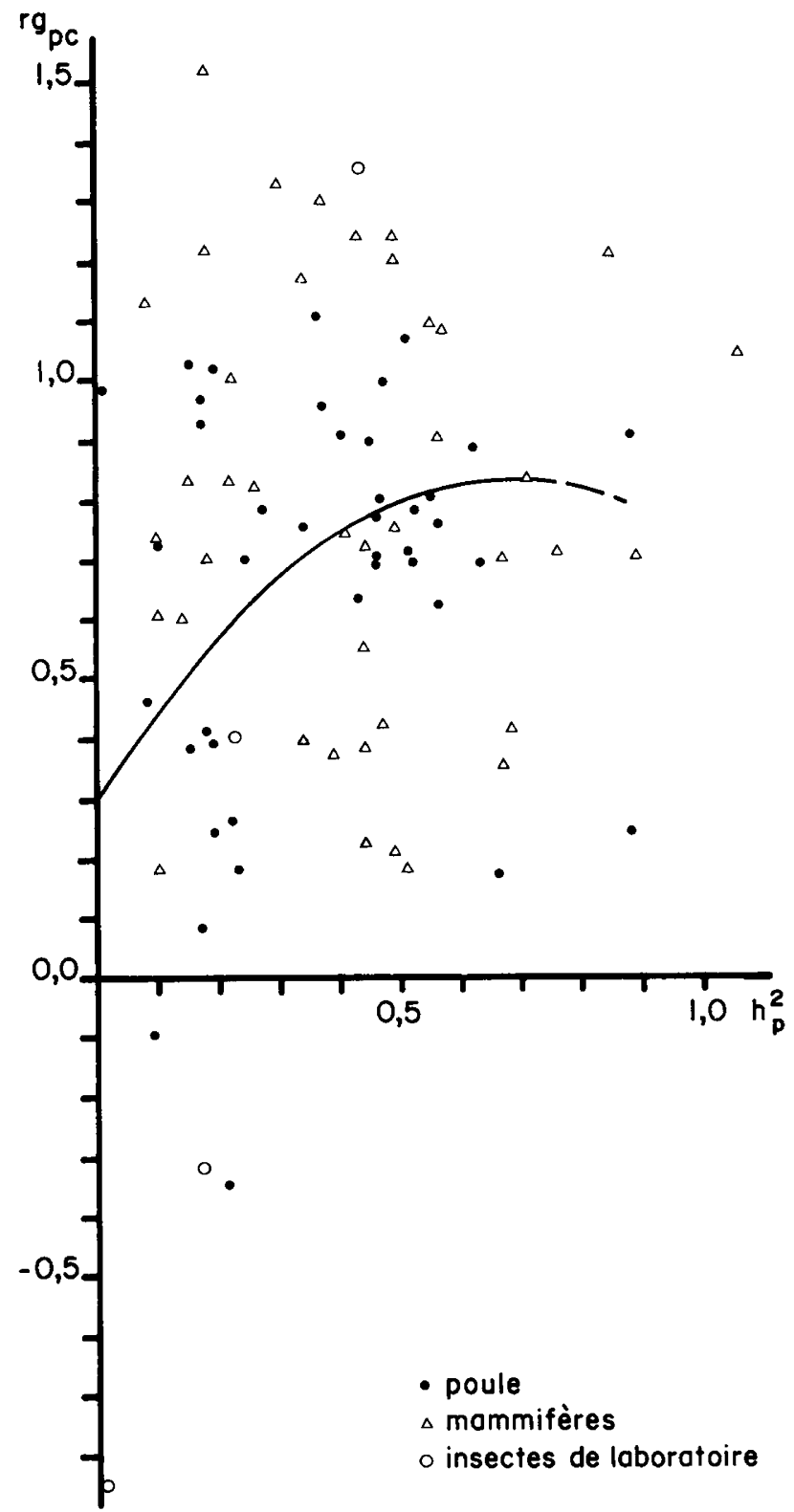

FIGURE 2

Estimées de la corrélation génétique pur-croisement $\left(\mathrm{rg}_{\mathrm{pc}}\right)$, rapportées aux valeurs correspondantes de $\mathrm{h}_{\mathrm{p}}^{2}$.

Estimates of the genetic correlation between purebred and crossbred performances $\left(\mathrm{rg}_{\mathrm{pc}}\right)$ plotted against the corresponding values for $\mathrm{h}_{\mathrm{p}}^{2}$. 


\section{Variations intra-espèce et caractère des indicateurs de l'interaction}

Plusieurs expériences de sélection récurrente et réciproque s'accompagnent d'une diminution de la corrélation génétique pur-croisement. PIrchner \& voN Krosigk (1973) rapportent une régression négative de $r g_{p c}$ sur le numéro de génération pour la production d'œufs dans 2 souches de pondeuses $(b=-0,11$ et $-0,16)$. HartmanN (1963) note une chute de cette corrélation de 0,66 à 0,25 en quelques générations de sélection sur le poids corporel dans des souches de poulet de chair. Ce phénomène est retrouvé par Mc New \& Bell (1974) sur le poids larvaire de Tribolium. Ainsi, la valeur de la corrélation génétique pur-croisement serait liée au degré de différenciation génétique des 2 souches, dans la mesure où la sélection récurrente et réciproque différencie génétiquement les souches en présence. Dans ce sens, on a recherché une liaison entre $r g_{p c}$ et l'hétérosis, sans pouvoir cependant la mettre en évidence. L'existence de différences de corrélation génétique pur-croisement selon le sens du croisement suggère l'intervention de facteurs liés à la souche d'origine des géniteurs testés en pur et en croisement : Hale \& Clayton (1965) remarquent, dans 2 souches de poule contrôlées sur la ponte, que la corrélation phénotypique entre moyennes de descendances pure et croisée de géniteurs d'une souche (sous estimation de $r g_{p c}$ ) est d'autant plus grande que l'héritabilité du caractère dans cette souche est plus grande. TARAN et al. (1972) expliquent quant à eux la différence de corrélation génétique pur-croisement qu'ils observent dans le croisement de 2 souches de pondeuses par leur différence d'histoire sélective, la plus faible valeur de $r g_{p c}$ correspondant à la souche la plus longuement sélectionnée. Le fait que des souches au plateau de sélection conduisent à des valeurs négatives de $r g_{p c}$ (BRown \& BELL, 1980, sur Drosophile) va dans le sens de l'hypothèse précédente.

\section{Analyse des paramètres * réalisés » des interactions géniteur $\times$ population partenaire à partir d'expériences de sélection}

\section{A. Méthode}

Soient 2 populations pures, les 2 populations issues de leurs croisements réciproques et soient 2 types de méthodes de sélection, la sélection intra-population et la sélection en croisement. Etant donnée une méthode de sélection, soit $\Delta \mathbf{P}$ la réponse moyenne des 2 populations pures et $\Delta \mathrm{C}$, celle des 2 populations croisées ${ }^{(1)}$. Si l'on désigne par $b_{c / p}$ le coefficient de régression de $\Gamma_{c}$ sur $\Gamma_{p}$ et par $b_{p / c}$ celui de la régression réciproque, on peut montrer (BRUN, 1984) que :

$$
\begin{aligned}
& \text { le rapport } \frac{\Delta \mathrm{C}}{\Delta \mathrm{P}} \text { en sélection intra-population estime } b_{c / p} \text { et } \\
& \text { le rapport } \frac{\Delta \mathrm{P}}{\Delta \mathrm{C}} \text { lors de sélection en croisement estime } b_{p / c}
\end{aligned}
$$

(1) En sélection intra-population $\Delta \mathrm{P}$ et $\Delta \mathrm{C}$ sont des réponses respectivement " directe " et " corrélée ». C'est l'inverse pour une sélection en croisement. 
Les coefficients $b_{c / p}\left(\right.$ ou $\left.b_{p / c}\right)$ ainsi calculés sont des coefficients de régression " réalisés ». Ils sont fonction des 2 paramètres $r g_{p c}$ et $\sigma=\left\{v\left(\Gamma_{c}\right) / v\left(\Gamma_{p}\right)\right\}^{0,5}$ avec $b_{c / p}=r g_{p c} \cdot \sigma$ et $b_{p / c}=r g_{p c /} \sigma$.

La connaissance simultanée de $b_{c / p}$ et $b_{p / c}$ est nécessaire pour calculer ces paramètres. La connaissance d'un seul de ces coefficients permet seulement de conclure, et encore s'il est différent de 1 , à l'existence d'interaction. Cependant, une estimation statistique de l'un des 2 paramètres de l'interaction $\left(r g_{p c}\right.$ ou $\left.\sigma\right)$ permettra d'accéder à l'autre.

Les expériences analysées sont classées au tableau 2 en fonction de la valeur du coefficient $b_{c / p}$ pour les expériences de sélection intra-population et du coefficient $b_{p / c}$ pour les expériences de sélection en croisement, en distinguant 3 classes de valeurs de $b$ :

1) le cas où $b=1$, correspondant à une réponse corrélée dont le profil est parallèle à celui de la réponse directe ;

2) le cas où $0 \leqslant b<1$, caractérisé par une réponse corrélée positive mais inférieure à la réponse directe ;

3) le cas où $b<0$, reflétant une réponse corrélée négative.

La seule valeur de $b$ supérieure à 1 rencontrée $\left(b_{c / p}=1,13\right.$ pour le poids larvaire dans les souches de type $C_{2}$ de Tribolium de Mc New \& Bell, 1976) est portée au tableau 2 dans la classe définie par $b=1$. Toute expérience présentant une évolution de $b$ au cours des générations est décomposée en expériences élémentaires où $b$ peut être considéré comme stable. On distingue ainsi 9 expériences de sélection intrapopulation et 14 expériences de sélection en croisement, constituées d'une majorité d'expériences de sélection récurrente et réciproque (S.R.R.).

L'examen des coefficients de régression "réalisés " permettra une première approche globale des interactions géniteur $\times$ population partenaire, visant surtout à les mettre en évidence. Il sera complété par une approche plus analytique visant à estimer les 2 paramètres réalisés de l'interaction soit en s'appuyant sur l'estimation statistique de l'un de ces 2 paramètres, soit en confrontant les coefficients $b_{c / p}$ et $b_{p / c}$ réalisés sur les mêmes populations (cellules encadrées du tabl. 2).

\section{B. Variations de $b_{c / p}$ (sélection intra-population)}

Ce coefficient de régression est voisin de 1 dans 7 cas sur 9 et prend, dans les 2 autres cas, une valeur inférieure à 1 mais positive. On ne peut conclure d'emblée à l'existence d'interaction que dans ces 2 derniers cas. L'un concerne les 10 dernières générations d'une expérience de sélection sur le poids pupal chez Tribolium (WoNG \& Boylan, 1970) avec un coefficient $b_{c / p}$ voisin de 0 . Il reflète une absence de réponse corrélée en croisement alors que la réponse directe se maintient jusqu'à la fin de l'expérience, à la génération 22 . Sur l'ensemble de l'expérience de coefficient $b_{c / p}$ passe de 1 à 0 , avec une valeur globale de l'ordre de 0,70 . Cette expérience nous fournit en outre une estimation statistique moyenne de la corrélation génétique pur-croisement $\left(r g_{p c}=0,40 \pm 0,17\right)$ d'où découle une valeur de $\sigma$ de 1,75 . La diminution de $b_{c / p}$ peut résulter d'une diminution de $r g_{p c}$ (qui passerait de 0,6 à 0 à $\sigma$ constant) ou de $\sigma$, qui tendrait alors vers 0 . L'autre cas d'interaction concerne le poids larvaire chez Tribolium (Mc New \& BeLl, 1976) avec un coefficient $b_{c / p}$ égal à 0,6 pour une expérience de 11 


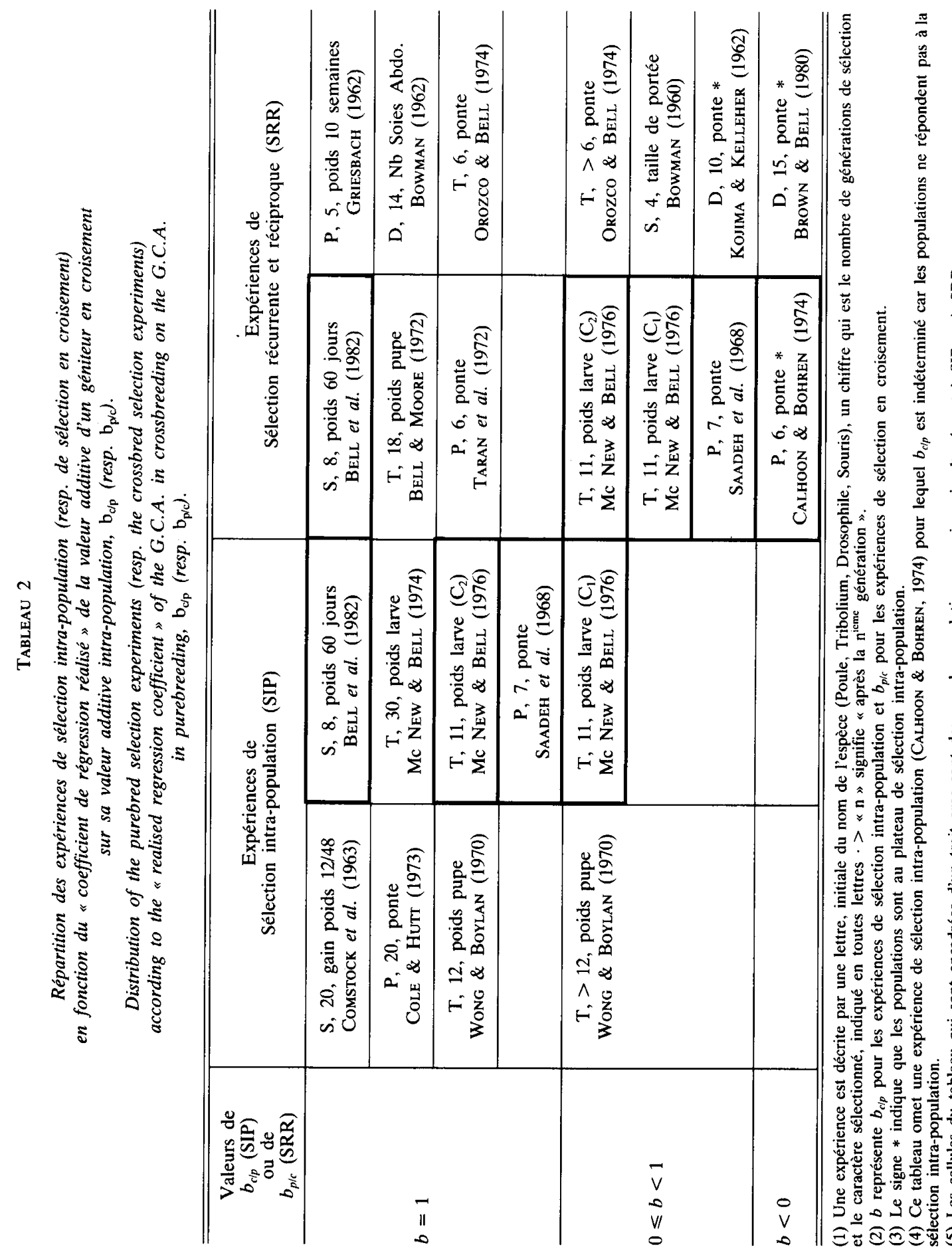


générations. Au cours d'une expérience antérieure sur 30 générations, les mêmes populations avaient réalisé un coefficient de régression égal à 1 (Mc New \& BeLL, 1974). Si l'évolution de $b_{c / p}$ rappelle celle observée précédemment sur le poids pupal, il serait abusif d'assimiler les 2 résultats : les 2 phases de l'expérience de sélection sur le poids larvaire sont séparées par 5 générations de relâchement de la sélection, dont l'effet dépressif sur la moyenne du caractère a été significativement plus sensible sur les populations pures que sur leurs croisements. La supériorité du progrès ultérieur chez les purs n'en serait peut-être que la contrepartie.

Notons enfin l'absence de valeüs négatives de $b_{c / p}$ et donc de la corrélation génétique pur-croisement.

\section{Les variations de $b_{p / c}$ (sélection en croisement)}

Les valeurs du coefficient de régression pur/croisement sont plus diversifiées que celles de la régression inverse : sur les 14 expériences que nous avons distinguées, 8 correspondent à des valeurs inférieures à 1 , dont 2 à des valeurs négatives. Ces 8 cas, qui signalent l'existence d'interactions, concernent des caractères de productivité numérique à l'exception de 2 , relatifs au poids larvaire chez Tribolium (Mc New \& BELL, $1976, C_{1}$ et $C_{2}$ ). Pour ce dernier caractère, le coefficient de régression pur/croisement réalisé est de 0,37 dans le cas où les populations de base résultent directement d'un programme de 30 générations de sélection intra-population (constitution génétique $\mathrm{C}_{1}$ ) et de 0,69 lorsque les populations de base sont constituées par inter-croisements suivis de panmixie entre les populations préalablement sélectionnées (constitution génétique $\mathrm{C}_{2}$ ).

Dans le cas des souches de Tribolium sélectionnées sur la ponte par Orozco \& BELL (1974) dans 3 ambiances thermiques différentes, des interactions apparaissent vers la $7^{\mathrm{e}}$ génération de sélection récurrente et réciproque avec une divergence entre les réponses des populations pures et croisées et une régression pur/croisement de l'ordre de 0,7 . Cette diminution de la régression pur/croisement à partir de 1 pourrait s'expliquer par une augmentation de $\sigma$ dans les 2 milieux thermiques les plus défavorables à la ponte ${ }^{(1)}$, et par une diminution de la corrélation génétique pur-croisement dans le milieu optimum, où les héritabilités en pur et en croisement restent égales.

Les expériences de SAadeh et al. (1968) et Calhoon \& Bohren (1974) chez la poule, marquées par des valeurs respectivement faibles $\left(b_{p / c}=0,15\right)$ et négative $\left(b_{p / c}=-2\right)$ de la régression pur-croisement pour la ponte, impliquent également des interactions. Un autre cas d'interaction concerne une expérience de sélection récurrente à testeur constant pour augmenter la taille de portée dans une souche de souris (Bowman, 1960) : on constate une augmentation de la taille de portée des femelles croisées sans augmentation corrélative de la taille de portée intra-population. Ce cas correspond à une corrélation génétique pur-croisement réalisée qui est nulle, en accord avec les valeurs estimées des covariances génétiques pur-croisement, prenant, sur 4 générations, 3 valeurs négatives et une valeur positive.

(1) Sur l'ensemble de l'expérience, on peut, sur la base de $h_{c}^{2} / h_{p}^{2}$, estimer $\sigma$ à 1,25 alors qu'il est initialement de 1 puisque les populations de base dérivent d'une même population par simple subdivision. 
Enfin, l'expérience de S.R.R. de Brown \& BeLl (1980) sur la Drosophile conduit à une réponse corrélée négative des populations parentales sélectionnées pour la ponte pendant 15 générations. Les populations de base sont à un plateau lié à une extinction de la variance génétique additive intra-population. La réponse corrélée négative $\left(b_{p / c}=-0,54\right)$ est en accord avec les estimées des corrélations génétiques pur-croisement en début de l'expérience qui sont de $-0,85 \pm 0,70$ et $-0,35 \pm 0,25$ pour les 2 souches respectivement.

Remarquons enfin un cas de profil de réponse corrélée assez inattendu (KoJima \& Kelleher, 1962), caractérisé par une phase initiale de latence où les populations parentales ne répondent pas, avant une phase de réponse positive alors que la réponse directe en croisement est immédiate. Ce cas correspond à une évolution de la covariance génétique pur-croisement d'abord nulle puis positive, vraisemblablement liée à une absence de variation additive libre intra-population en début d'expérience $\left[v\left(\Gamma_{p}\right)=0, \operatorname{cov}\left(\Gamma_{p}, \Gamma_{c}\right)=0\right.$ et $\left.v\left(\Gamma_{c}\right)>v\left(\Gamma_{p}\right)\right]$ puis à une libération de variation additive intra-population. Il indique encore la possibilité d'une évolution des paramètres de l'interaction au cours d'un processus de sélection et d'une non linéarité de la liaison pur-croisement.

Toutes les expériences telles que $b_{p / c} \simeq 1$ correspondent vraisemblablement à une absence d'interaction géniteur $\times$ population partenaire compte tenu : (i) de la valeur estimée de $\sigma(\sigma \simeq 1)$ dans les expériences de Bell \& Moore (1972), Orozco \& Bell (1972) et Bowman (1962) ; (ii) des résultats généraux de la bibliographie (indiquant une faible probabilité chez la poule que $h_{p}^{2}$ soit supérieur à $h_{c}^{2}$ et donc $\sigma \geqslant 1$ ) pour les expériences de Griesbach (1962) et TARAN et al. (1972) ; (iii) de la connaissance du coefficient de la régression inverse $\left(b_{p / c} \simeq 1\right)$ chez BELl et al. (1982). Dans le cas de Bowman (1962) et de l'une des 2 souches utilisées par TARAN et al. (1972), ces conclusions sont confirmées par l'estimation statistique de $r g_{p c}\left(r g_{p c}=1,35\right.$ et 1,02 resp.).

\section{Analyse de l'interaction à partir de la connaissance simultanée de $b_{c / p}$ et $b_{p / c}$}

Dans 5 cas, la connaissance simultanée des 2 coefficients de régression $b_{c / p}$ et $b_{p / c}$ permet d'estimer les composantes de l'interaction (tabl. 3). Les interactions signalées dans les souches de Tribolium sélectionnées sur le poids larvaire (Mc NEw \& BeLL, 1976) se caractérisent par une valeur de $\sigma$ supérieure à 1 et apparemment indépendante de la constitution génétique des souches. Par contre, la corrélation génétique purcroisement réalisée est plus élevée pour les souches néo-formées par synthèse entre souches préalablement sélectionnées que pour ces souches elles-mêmes qui ont un long passé de sélection artificielle intra-population.

L'expérience de SAADEH et al. (1968) conduit également à des interactions du même type. La discordance entre ces valeurs "réalisées » et les estimations statistiques en début d'expérience $\left(r g_{p c} \simeq 1\right.$ et $\left.\sigma \simeq 1\right)$ indique une diminution de $r g_{p c}$ et une augmentation de $\sigma$ en cours de S.R.R.

Dans l'expérience de Calhoon \& Bohren (1974), l'absence de réponse à la sélection intra-population indique qu'on est à un plateau de sélection, impliquant soit une extinction de la variabilité additive intra-population $\left(\nu\left(\Gamma_{p}\right) \simeq 0\right)$ soit l'existence de 


\section{TABLEAU 3}

Estimation des paramètres de l'interaction

à partir des expériences fournissant simultanément $\mathrm{b}_{\mathrm{c} / \mathrm{p}}$ et $\mathrm{b}_{\mathrm{p} / \mathrm{c}}$.

Estimation of the parameters of the interaction

from the experiments providing both $\mathrm{b}_{\mathrm{c} / \mathrm{p}}$ and $\mathrm{b}_{\mathrm{p} / \mathrm{c}}$.

\begin{tabular}{|c|c|c|c|c|c|}
\hline & & $b_{c / p}$ & $b_{p / c}$ & $r g_{p c}$ & $\sigma$ \\
\hline \multirow{2}{*}{$\begin{array}{l}\text { Poids larvaire } \\
\text { Tribolium } \\
\text { Mc New \& BeLl (1976) }\end{array}$} & Souches $C_{1}$ (a) & 0,61 & 0,37 & 0,47 & 1,30 \\
\hline & Souches $C_{2}$ (a) & 1,13 & 0,69 & 0,88 & 1,30 \\
\hline $\begin{array}{l}\text { Poids à } 60 \text { jours } \\
\text { Souris } \\
\text { BELL et al. }(1982)\end{array}$ & & 1 & 1 & 1 & 1 \\
\hline $\begin{array}{l}\text { Ponte } \\
\text { Poule } \\
\text { CALHOON \& BOHREN (1974) }\end{array}$ & & $\begin{array}{c}\text { indéterminé } \\
v\left(\Gamma_{p}\right) \simeq 0 \\
\text { (Plateau de } \\
\text { sélection) }\end{array}$ & -2 & $\begin{array}{l}\text { indéterminé } \\
\qquad 0\end{array}$ & 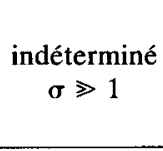 \\
\hline $\begin{array}{l}\text { Ponte } \\
\text { Poule } \\
\text { SAADEH et al. (1968) }\end{array}$ & & 1 & 0,15 & 0,4 & 2,5 \\
\hline
\end{tabular}

(a) : $C_{1}$ et $C_{2}$ désignent des types de souches : les souches $C_{1}$ résultent directement d'un programme de sélection intra-population, les souches $C_{2}$ proviennent de croisements synthétiques entre les souches $C_{1}$.

pressions antagonistes à la sélection. L'observation des réponses à la S.R.R. (réponse positive en croisement et négative intra-population) indique l'existence d'une variabilité additive non nulle en croisement et une covariance génétique pur-croisement négative, au moins sur les 3 derniers cycles de sélection. On serait dans un cas d'interaction impliquant ses 2 composantes. Cependant, il faut nuancer cette interprétation du fait de l'existence de consanguinité (le coefficient moyen de consanguinité des souches sélectionnées augmente en moyenne de 2 p. 100 par génération) et d'interactions éventuelles entre sélection et consanguinité (CALHOon \& BoHren, 1974).

\section{E. Synthèse}

Les 17 couples de populations étudiés se répartissent en 9 cas où l'on a pu mettre en évidence une interaction entre géniteur et population partenaire, 4 cas de noninteraction et 4 cas pour lesquels on ne peut pas conclure. Les 9 cas d'interaction sont représentés par 6 caractères de reproduction ( 1 chez la souris et Tribolium, 2 chez la poule et la Drosophile) et 3 caractères pondéraux, dont 2 présentent de l'hétérosis (poids larvaire chez Tribolium, souches $C_{1}$ et $C_{2}$ ) et l'autre non. Les 4 cas de noninteraction concernent 3 caractères pondéraux (poids à 60 jours chez la souris et à 10 semaines chez le poulet de chair, poids pupal chez Tribolium), le quatrième étant la ponte chez la poule. La fréquence des situations d'interaction dans l'échantillon étudié 
est ainsi plus forte pour les caractères présentant de l'hétérosis. De plus, ces interactions apparaissent surtout lors de sélection en croisement : dans les 3 cas où les 2 types de méthodes sont appliqués, la sélection récurrente et réciproque exprime mieux ces interactions dans le sens où l'écart entre réponses directe et corrélée y est plus grand.

\section{Discussion générale}

En dépit de ses limites, liées à la rareté des résultats expérimentaux et à l'imprécision statistique des paramètres de l'interaction, cette étude révèle des tendances générales que l'on retrouve dans les différentes espèces étudiées et à travers les 2 démarches utilisées pour étudier l'interaction, l'estimation des paramètres et l'analyse des expériences de sélection : l'interaction semble affecter préférentiellement les caractères à faible héritabilité et présentant de l'hétérosis, comme ceux d'adaptation et notamment de reproduction. Elle se traduit de façon concordante au niveau des paramètres estimés par une supériorité de l'héritabilité en croisement et une faible valeur de la corrélation génétique pur-croisement et au niveau des expériences de sélection récurrente et réciproque par une faible corrélation réalisée entre les réponses intra-population et en croisement. On note cependant des cas d'interaction sur des caractères pondéraux, à plus forte héritabilité, notamment sur les poids à la naissance et au sevrage chez les ovins et bovins, voire à un stade plus tardif du développement chez les mammifères polytoques (souris et porc). Dans ce cas, l'interaction se traduit préférentiellement par des variabilités génétiques additives supérieures en race pure. Le fait que de telles interactions n'existent pas chez la poule, espèce où la présence maternelle auprès des jeunes est relativement moins longue que chez les mammifères qui allaitent leurs jeunes suggère l'intervention d'influences maternelles dans leur déterminisme. L'opposition généralement admise entre les caractères de production et de reproduction se retrouverait ainsi dans la façon dont se manifestent les interactions étudiées.

L'histoire sélective des souches en présence, en particulier de la souche donneuse des géniteurs testés en pur et en croisement, semble jouer un rôle important dans le déterminisme de ces interactions. Cela ressort de l'étude des paramètres génétiques (chez la poule et la drosophile) et surtout des expériences de sélection en croisement conduites sur des populations après un grand nombre de générations de sélection intrapopulation voire au plateau de sélection. Dans la majorité de ces cas, constituée par des caractères présentant de l'hétérosis, elles apparaissent comme la forme exacerbée des interactions observées sur les mêmes caractères moins sélectionnés : variabilité additive supérieure en croisement et corrélation génétique pur-croisement faible voire négative. Un long passé de sélection artificielle peut également générer des interactions sur des caractères sans hétérosis, comme le poids pupal chez Tribolium, avec dans ce cas une variabilité additive en croisement qui peut décroître pour devenir inférieure à celle qui existe intra-population.

Ces différences de variabilité génétique en pur et en croisement peuvent s'interpréter dans le cadre d'un modèle simple de génétique quantitative comme le modèle à 1 locus biallélique. Ce modèle peut générer, en cas de superdominance, des valeurs négatives de la corrélation génétique pur-croisement (BowmAN, 1960). Il peut également rendre compte de différences de variabilités additives en pur et en croisement, et en 
particulier d'une situation où le croisement restaure une variabilité génétique additive dont la valeur est nulle, intra-population (équilibre de superdominance). Dans ce cas, toute la variance génétique intra-population est de dominance et BELL (1982) interprète l'augmentation de variabilité additive lors du croisement comme une transformation de variabilité non-additive intra-population. Cependant, un changement du mode d'expression des gènes en fonction de leur contexte génétique pourrait également expliquer ces différences de variabilité génétique selon le système de reproduction. C'est une hypothèse avancée par Gallais $(1972$, 1984) pour expliquer la coexistence d'additivité en croisement et de non-additivité en régime de consanguinité observée chez la luzerne. Un tel effet de la structure génétique (homozygotie ou hétérozygotie) sur l'expression de la variabilité a été observée par MuKaI et al. (1966) chez la Drosophile.

L'étude des expériences de sélection met en évidence une dissymétrie de la régression pur-croisement, se traduisant, pour les caractères d'adaptation présentant de l'interaction, par le fait d'un gain sur la valeur génétique additive intra-population est généralement répercuté en croisement alors que l'inverse n'est pas vrai. Jointe à l'observation d'un profil curvilinéaire de la réponse intra-population dans certains cas de sélection récurrente et réciproque, cette dissymétrie peut s'interpréter par l'existence d'une liaison non linéaire entre les effets additifs d'un géniteur intra-population d'une part $\left(\Gamma_{p}\right)$ et en croisement d'autre part $\left(\Gamma_{c}\right)$. Un tel schéma illustre également le rôle joué dans le "déterminisme » des interactions étudiées par la sélection artificielle, en expliquant les réponses corrélées négatives en pur sur des populations au plateau de sélection. De plus, il rend compte des différences de variabilités génétiques additives en pur et en croisement pour les caractères d'adaptation : celle qui existe intra-population apparaît comme "bridée " relativement à son expression en croisement, d'où des héritabilités supérieures en croisement.

\section{Conclusion}

L'objet de cette étude était d'apporter une réponse en termes biologiques à la question du choix entre 2 méthodes de sélection pour améliorer le croisement, la sélection intra-population et la sélection récurrente et réciproque. La réponse à cette question en termes de génétique quantitative apparaissait a priori difficilement envisageable, en rapport avec la prédiction du progrès par la $2^{\mathrm{e}}$ méthode, réputée utiliser une variabilité génétique non-additive. Les statistiques que l'on a proposées pour paramétrer les interactions entre géniteurs et populations partenaires (variance et covariance des A.G.C. en pur et en croisement) rejoignent en dernière analyse les paramètres génétiques classiques (corrélation génétique pur-croisement, héritabilités en pur et en croisement) ; c'est d'ailleurs pourquoi l'on a adopté cette terminologie pour les paramètres de l'interaction. Sur le plan appliqué, les conclusions de cette étude n'apportent rien par rapport à celles de A.E. BELL (1982) dont l'approche est restée dans le droit fil de la génétique quantitative. L'apport de cette étude est peut-être une vision synthétique de 2 phénomènes, l'écart à 1 de la corrélation génétique pur-croisement et la différence d'héritabilité en pur et en croisement qui varient de façon conjointe avec le déterminisme génétique du caractère. De plus cette étude atteste l'existence d'un phénomène biologique mal étudié à savoir une différence d'expression du génotype d'un même géniteur selon la population partenaire. Il s'agit d'une extension des 
interactions "épistatiques " existant entre un gène et son environnement génétique. La synthèse de Hallauer \& Miranda (1981) sur les méthodes de sélection récurrentes appliquées au maïs indique clairement l'existence de phénomènes analogues chez les plantes.

Reçu le 6 mai 1983.

Accepté le 6 mars 1985.

\section{Remerciements}

L'auteur remercie cordialement A.E. Bell (Purdue University, Lafayette, U.S.A.) et F. Orozco (Instituto nacional de Investigationes Agrarias, Madrid, Espagne) pour leurs fructueux enseignements ainsi que les personnes qui ont participé à la mise en forme du manuscrit, en particulier les lecteurs anonymes mandatés par la revue, l'éditeur scientifique ainsi qu'A. GiBon et $R$. ROUVIER.

\section{Références bibliographiques}

Basset J.W., Shelton M., 1966. Crossbreeding influence on heritability estimates. J. Anim. Sci., 25, 877 (Abstr.).

Bell A.E., Moore C.H., 1972. Reciprocal recurrent selection for pupal weight in Tribolium in comparison with conventional methods. Egypt. J. Genet. Cytol., 1, 92-119.

Bell A.E., Gajic Z., Harrington R.B., 1982. Comparison of crossbred and purebred selection for 60 day body weight in two long term selected mouse populations (non publié, cité in BELL, 1982).

Bell A.E., 1982. Utilization of non additive gene effects. Experimental results from laboratory and domestic animals. Les Colloques de l'I.N.R.A. $n^{\circ}$ 10, I.N.R.A. Publ. (éd.), Toulouse, 13 . 14 octobre 1981, 107-123.

Biswas D.K., Craig J.V., 1969. Relationship between purebred and crossbred paternal half-sisters' performance in chickens. Poult. Sci., 48, 524-526.

Biswas D.K., Chapman A.B., First N.L., Self H.L., 1971. Intrapopulation versus reciprocal selection in Swine. J. Anim. Sci., 32, 840-848.

Bowman J.C., 1962. Recurrent selection. II. An experimental study with mice and Drosophila. Genet. Res., 3, 333-351.

Bowman J.C., 1972. Genotype $\times$ environment interactions. Ann. Génét. Sél. Anim., 4, 117-123.

Brown W.P., Bell A.E., 1980. An experimental comparison of selection alternatives to plateaued response. Genetics, 94, 477-496.

BRUN J.M., 1982. Interactions géniteur $\times$ population des partenaires. I. Définition d'indicateurs. Ann. Génét. Sél. Anim., 14, 463-479.

BRUN J.M., 1984. Interactions géniteur $\times$ population des partenaires. II. Détection par des expériences de sélection. Génét. Sél. Evol., 16, 455-466.

Calhoon R.E., Bohren B.B., 1974. Genetic gains from reciprocal recurrent and within line selection for egg production in the fowl. Theor. Appl. Genet., 44, 364-372.

CAMPO J.L., 1982. Reciprocal recurrent selection in layers : the significance of correlations between purebred and crossbred progeny. Les Colloques de l'I.N.R.A. $n^{o}$ 10, I.N.R.A. Publ. (éd.), Toulouse, 13-14 octobre 1981, 83-88.

Cole R.K., Hutt F.B., 1973. Selection and heterosis in Cornell white Leghorns : a review with special consideration of interstrain hybrids. Anim. Breed. Abstr., 41, 103-118. 
Comstock R.E., Singh M., Enfield F.D., 1963. Selection for growth in mice : crossbred performance. J. Anim. Sci., 22, 1109 (Abstr.).

Dickerson G.E., 1952. Inbred lines for heterosis tests ? In: Gowen J.W. (ed.), Heterosis, 330351. Iowa Stade College Press, Ames, Iowa.

DicKerson G.E., 1962. Implications of genetic environmental interaction in animal breeding. Anim. Prod., 4, 49-63.

Dunn R.J., Magee W.T., Gregory K.G., CundifF L.V., Koch R.M., 1970. Genetic parameters in straightbred and crossbred beef cattle. J. Anim. Sci., 31, 656-663.

Emsley A., Dickerson G.E., Kashyap T.S., 1977. Genetic parameters in progeny test selection for field performance of strain-cross layers. Poult. Sci., 56, 121-146.

ENFIELd F.D., Rempel W.E., 1962. Covariance of sire effects in purebred and crossbred populations of Swine. J. Anim. Sci., 21, 971 (Abstr.).

Gallais A., 1977. Contribution à l'étude théorique et expérimentale de l'hétérosis chez une plante allogame antotétraploïde. Thèse de doctorat. Université de Paris Sud.

Gallais A., 1984. An analysis of heterosis vs. inbreeding effects with an autotetraploid crossfertilized plant : Medicage Sativa L. Genetics, 106, 123-137.

Griesbach L., 1962. A test of the reciprocal recurrent selection technique with chicken broilers. Can. J. Anim. Sci., 42, 145-157.

Hale R.W., Clayton G.A., 1965. A diallel crossing experiment with two breeds of laying fowls. Brit. Poult. Sci., 6, 153-174.

Hallauer A.R., Miranda J.B., 1981. Quantitative Genetics in Maize Breeding. 468 pp., Iowa State University Press, Ames.

Hansson A., Lindkvist G., 1962. Selection for general and specific combining ability with regard to body weight in mice. Ann. Roy. Agric. Coll. Sweden, 28, 215-233.

Koger M., Jilex A.F., Burns W.C., Crokett J.R., 1975. Sire effects for specific combining ability in purebred and crossbred cattle. J. Anim. Sci., 40, 230-239.

KoJima K., Kelleher R.H., 1963. A comparison of purebred and crossbred selection schemes with two populations of Drosophila pseudo-obscura. Genetics, 48, 57-72.

Krause E., Yamada Y., Bell A.E., 1965. Genetic parameters in two populations of chickens under reciprocal recurrent selection. Brit. Poutl. Sci., 6, 197-206.

Louca A., Robison O.W., 1967. Components of variance and covariance in purebred and crossbred Swine. J. Anim. Sci., 26, 267-273.

Mc New R.W., Bell A.E., 1974. Crossbred responses from purebred selection ; an experimental check on selection theory with Tribolium. Theor. Appl. Genet., 44, 100-105.

Mc New R.W., Bell A.E., 1976. Comparison of crossbred and purebred selection for a heterotic trait in highly selected populations of Tribolium. J. Hered., 67, 275-283.

Mergl R., 1977a. Genetische Zusammenhänge zwischen Leistungen von Reinzucht und Kreuzungshennen. I. Genetische Parameter für Zuchthennen. Arch. Geflügelkd., 41, 92-97.

Mergl R., 1977b. Genetische Zusammenhänge zwischen Leistungen von Reinzucht und Kreuzungshennen. II. Genetische Parameter für Test-Kreuzungshennen. Arch. Geflügelkd., 41, 145148.

Mergl R., 1977c. Genetische Zusammenhänge zwischen Leistungen von Reinzucht und Kreuzungshennen. III. Genetische Korrelationen zwischen Legeleistung von Hybriden und Legeleistung und Fruchtbarkeitsmerkmalen von Reinzuchthennen. Arch. Geflügelkd., 41, 196-201.

Mukai T., Yowhikawa I., SANo K., 1966. The genetic structure of natural populations of Drosophila melanogaster. IV. Heterozygous effects of radiation induced mutations on viability in various genetic backgrounds. Genetics, 53, 513-527.

Orozco F., Bell A.E., 1974. Reciprocal recurrent selection compared to within strain selection for increasing rate of egg lay of Tribolium under optimal and stress conditions. Genetics, 77, 143-161.

Orozco F., Campo J.L., 1975. A comparison of purebred and crossbred genetic parameters in layers. World's Poult. Sci. J., 31, 149-153.

Orozco F., 1982. Utilization of the overdominance variance. Les Colloques de l'I.N.R.A. $n^{\circ} 10$, I.N.R.A. Publ. (éd.), Toulouse, 13-14 octobre 1981, 29-54. 
Pirchner F., von Krosigk G.M., 1973. Genetic parameters of cross and purebred poultry. Br. Poult. Sci., 14, 193-202.

Richardson R.H., KoJima K., 1965. The kinds of genetic variability in relation to selection response in Drosophila fecundity. Genetics, 52, 583-598.

Robison O.W., Louca A., Legates J.E., 1964. Purebred and crossbred performance of paternal half-sibs of swine. J. Anim. Sci., 23 (Abstr.).

SaAdeh H.K., Craig J.V., Smith L.T., Wearden S., 1968. Effectiveness of alternative breeding systems for increasing rate of egg production in chickens. Poult. Sci., 47, 1057-1072.

Salah E., Galal E., Hazel L.N., Sidwell G.M., Terril C.E., 1970. Correlation between purebred and crossbred half-sibs in sheep. J. Anim. Sci., 30, 475-480.

Sellier P., 1982. Selecting populations for use in crossbreeding. 2nd World Congress on Genetics applied to Livestock production, Madrid, 4-8 october 1982, 6, 15-49, Editorial Garsi, Madrid.

Standal N., 1968. Studies on breeding and selection schemes in pigs. I : Selection on performance of purebred versus crossbred progeny. Acta Agric. Scand., 18, 222-232.

Stanislaw C.M., Omtvedt I.T., Willham R.L., Whatley J.A. Jr., 1967. A study of some genetic parameters in purebred and crossbred populations of Swine. J. Anim. Sci., 26, 16-20.

Taran M., Gabriel E., Moses E., Soller M., 1972. Performance of purebred and crossbred progeny of white Leghorn and New-Hampshire sires. Br. Poult. Sci., 13, 331-339.

TAYlOR J.C., Miller R.H., Hetzer M.O., 1965. Genetic correlations between straightbred and crossbred swine. J. Anim. Sci., 24 (Abstr.).

Vinson W.E., EISEN E.J., RoBIson O.W., 1969. Predicted response to selection for crossbred performance in mice. J. Anim. Sci., 28, 725-733.

Wilson S.P., Whatley J.A. Jr., Whiteman J.V., Morrison R.D., 1962. Influences of sires and lines of breeding on sow productivity. J. Anim. Sci., 21, 119-122.

Wong W.C., Boylan W.J., 1970. Intrapopulation selection and correlated response in crossbred of Tribolium Castaneum. Genetics, 64, 59-68.

Wong W.C., Boylan W.J., Rempel W.E., 1971. Purebred versus crossbred performance as a basis of selection in swine. J. Anim. Sci., 32, 605-610. 